\title{
Parametrically coupled multiharmonic force imaging
}

M. Kurtuluş Abak, Ozan Aktas, Rashad Mammadov, İhsan Gürsel, and Aykutlu Dâna

Citation: Appl. Phys. Lett. 92, 223113 (2008);

View online: https://doi.org/10.1063/1.2940304

View Table of Contents: http://aip.scitation.org/toc/apl/92/22

Published by the American Institute of Physics

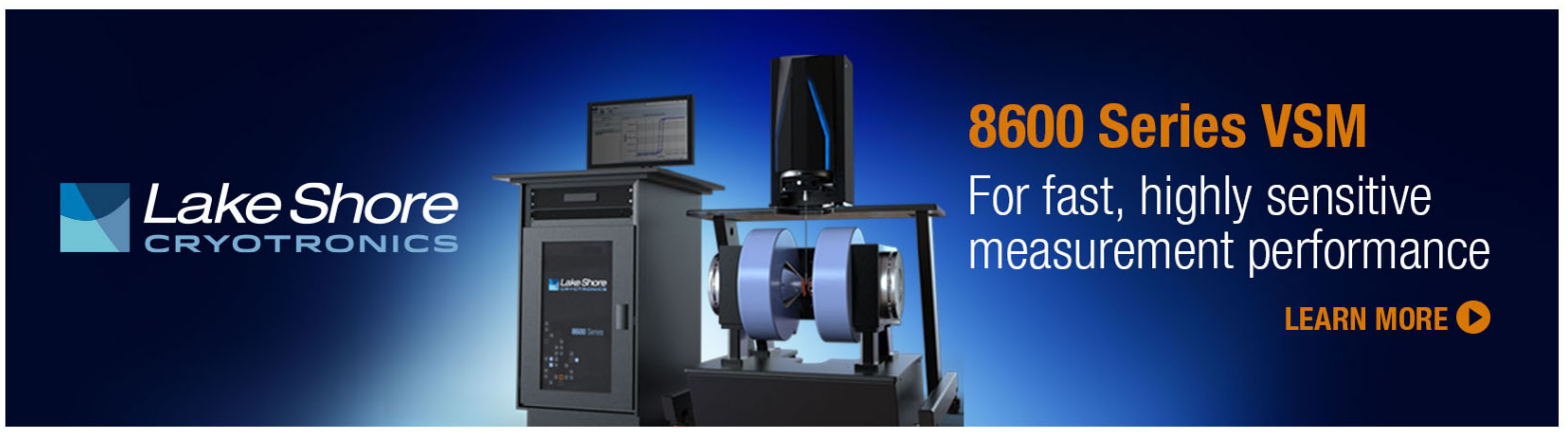




\title{
Parametrically coupled multiharmonic force imaging
}

\author{
M. Kurtuluş Abak, ${ }^{1}$ Ozan Aktas, ${ }^{2}$ Rashad Mammadov, ${ }^{1}$ İhsan Gürsel, ${ }^{1,3}$ and \\ Aykutlu Dâna ${ }^{1, a)}$ \\ ${ }_{1}^{1}$ Institute of Materials Science and Nanotechnology, Bilkent University, 06800 Ankara, Turkey \\ ${ }^{2}$ Department of Physics, Bilkent University, 06800 Ankara, Turkey \\ ${ }^{3}$ Department of Molecular Biology and Genetics, Bilkent University, 06800 Ankara, Turkey
}

(Received 19 February 2008; accepted 16 May 2008; published online 6 June 2008)

\begin{abstract}
We report use of nonlinear tip-sample interactions to parametrically convert the frequency components of periodic tip-sample interaction forces to frequencies where they can be resonantly detected. One flexural mode of a cantilever is used for tapping-mode imaging and another flexural mode is used for detection of forces converted in presence of an externally injected mechanical oscillation at the difference frequency of the detecting mode and a harmonic of the tapping mode. Material contrast in attractive and repulsive regimes are demonstrated on samples with polymethyl methacrylate patterns and with deoxyribonucleic acid strands on silicon. (c) 2008 American Institute of Physics. [DOI: 10.1063/1.2940304]
\end{abstract}

Measurement and imaging of material dependent nonlinear tip-sample interactions in atomic force microscopy ${ }^{1}$ (AFM) provides information about the structure of the sample on the nanoscale. In the conventional tapping mode, a single mode of the cantilever is used to resonantly detect the fundamental frequency component of the time varying interaction force, which is observed in the perturbations of the amplitude and phase of the oscillation. ${ }^{2}$ This selective measurement filters out higher harmonics of the interaction force and results in loss of information and material contrast is observed in dissipation related phase changes in the oscillation. $^{3-5}$ Although excitation of the higher frequency harmonics can be strongly related to material elasticity they are not detected in conventional tapping mode. ${ }^{6}$ The main reason for this is, for a rectangular cantilever, higher order mode frequencies are not integer multiples of the fundamental mode frequency. ${ }^{7-11}$ Several authors have addressed the problem of measuring the complete tip-sample interaction during tapping using special modifications to the cantilever or the scanning probe electronics. ${ }^{12,13}$ Multifrequency excitation of the cantilever simultaneously at the two flexural resonances has also been demonstrated to give complementary material contrast. ${ }^{14,15}$

In this article, the highly nonlinear tip-sample interaction is used to convert the frequency components of the interaction force generated during tapping to a frequency that can be resonantly measured. Both parametric up-conversion (PUC) and down-conversion (PDC) schemes can be implemented. One of the flexural modes is used in tapping mode imaging while a sinusoidal mechanical signal is applied at the difference frequency of the force component to be measured and the mode frequency to be used for detection. The frequency-converted force components are then resonantly detected using a lock-in amplifier. Frequency conversion efficiency is also related to the mechanical properties of the sample, therefore the conversion process itself also results in material contrast.

In the experiments different commercial AFM systems (PSIA XE100 retrofitted with a homemade vibrating sample holder and an Asylum MFP 3D system fitted with an external

\footnotetext{
${ }^{\text {a)} E l e c t r o n i c ~ m a i l: ~ a y k u t l u @ f e n . b i l k e n t . e d u . t r . ~}$
}

circuit for multifrequency excitation) are used. One of the flexural harmonics is used in tapping mode by the AFM controller and the ac deflection of the other flexural mode is detected using a rf lock-in amplifier (model SR844). Commercial rectangular AFM cantilevers are used. Typical measured mode resonant frequencies are $f_{0}=68-80 \mathrm{KHz}, f_{1}$ $=410-500 \mathrm{KHz}$, and nominal spring constants are $k_{0}$ $=2.7 \mathrm{~N} / \mathrm{m}, k_{1}=\left(f_{1} / f_{0}\right)^{2} k_{0}=99.3 \mathrm{~N} / \mathrm{m}$. Typical quality factors of the fundamental and second order flexural modes are determined to be $Q_{0} \simeq 200$ and $Q_{1} \simeq 500$. Supplier specified tip radius is $R=15 \mathrm{~nm}$. Deflection detection noise floor of the AFM systems used are $x_{\min } \sim 2 \times 10^{-13} \mathrm{~m} / \sqrt{\mathrm{Hz}}$ and resonant force measurements are Brownian motion limited. In PUC experiments, the fundamental mode is driven with an amplitude $A_{0} \simeq 10 \mathrm{~nm}$ at a frequency $f_{0}^{\prime}=f_{0}-\Delta f$, and a local oscillator vibration with amplitude $A_{\mathrm{LO}} \simeq 1 \mathrm{~nm}$ is applied either by vibrating the tip or the sample at a frequency $f_{\mathrm{LO}}$. The result of the mixing is monitored at $f_{1}$. Repulsive or attractive branches of the tip-sample interaction can be chosen by selecting a sufficiently large positive or negative frequency offset $\Delta f{ }^{16}$ The parametrically excited second order mode amplitudes $A_{1}$ as a function of the tip-sample separation $z_{\mathrm{ts}}$ are plotted in Figs. 1(b)-1(d) for $f_{\mathrm{LO}}=f_{1}-n f_{0}^{\prime}$ where $n=1,3,6$ (other $n$ not shown for figure clarity). The PUC process is seen to depend on local oscillator amplitude $A_{\mathrm{LO}}$ [Fig. 1(e)]. In the down-conversion process, the second order flexural mode is used in tapping and lever vibration is monitored at a frequency $f^{*}=f_{1}^{\prime}-f_{\mathrm{LO}}$. Since the fundamental mode spring constant $k_{0}$ is smaller compared to the higher order mode spring constant $k_{1}$, resonant frequency shift of the fundamental mode is more pronounced [Fig. 1(f)]. For resonant detection of PDC signal, $f_{\mathrm{LO}}$ was carefully chosen to coincide the PDC signal with the shifted fundamental mode resonance frequency $f_{0}^{\prime}$ [Fig. $1(\mathrm{~g})$ ]. However, nonresonant detection of PDC signal is also possible, at the expense of increased $A_{\mathrm{LO}}$ [Fig. 1(h)]. In all cases, in order to avoid jumping between attractive and repulsive regimes, $A_{\mathrm{LO}}$ must be kept adequately small. For certain set of parameters, instabilities are observed for very large $A_{\mathrm{LO}}(>5 \mathrm{~nm})$ and in some cases increased stability was observed with increasing $A_{\mathrm{LO}}$, possibly due to dithering effects. ${ }^{17}$ The noise spectrum 


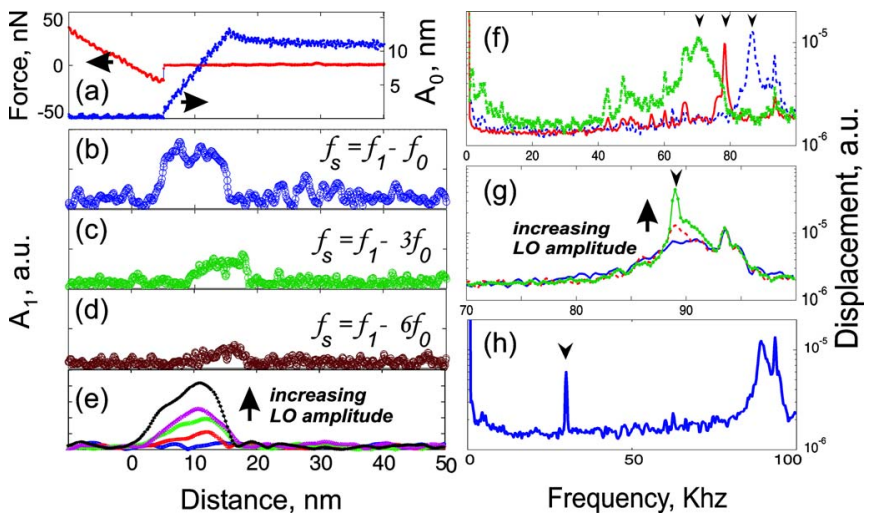

FIG. 1. (Color online) Excitation of the second order mode through parametric mode coupling as a function of tip-sample separation during tapping with the fundamental mode in attractive regime. The sample is $100 \mathrm{~nm}$ thick PMMA on silicon. (a) Typical force-distance and amplitude-distance curves acquired simultaneously with the PUC measurements. Second order mode amplitude $A_{1}$ when (b) $f_{\mathrm{LO}}=f_{1}-f_{d}$, (c) $f_{\mathrm{LO}}=f_{1}-3 f_{d}$, and (d) $f_{\mathrm{LO}}=f_{1}-6 f_{d}$. (e) Parametrically up-converted amplitude $A_{1}$ increases with increasing LO amplitude $A_{\mathrm{LO}}$. (f) Perturbation of the dynamic properties of the fundamental mode is observed in the noise spectrum of the cantilever deflection signal when the second mode is tapping in attractive (left arrow) and repulsive modes (right arrow) and away from the surface (middle arrow). (g) Parametrically down-converted signal at the shifted fundamental mode resonance frequency during tapping with higher order flexural mode. As $A_{\mathrm{LO}}$ is increased parametrically down-converted signal amplitude increases. (h) Parametrically down-converted signal to $30 \mathrm{KHz}$ (nonresonant).

of the phase signal was observed before measurements to ensure stability.

In order to understand the parametric conversion of force harmonics, we use numerical analysis. The cantilever dynamics can be modeled using lumped independent harmonic oscillators for different flexural modes. The resonant frequency shift of the cantilever mode is given by ${ }^{18}$

$$
\delta f\left(z_{\mathrm{ts}}\right)=\frac{f^{2}}{k A} \int_{0}^{1 / f} F_{\mathrm{ts}}\left[z_{\mathrm{ts}}+A \cos (2 \pi f t)\right] \cos (2 \pi f t) d t
$$

where $F_{\text {ts }}$ is the tip-sample interaction force, $A$ is the amplitude of oscillation, $k$ is the lumped spring constant, and $f$ is the resonant frequency. When the cantilever is driven by a fixed amplitude sinusoidal force $F_{d}(t)=F_{d} \cos \left(2 \pi f_{d} t\right)$ at frequency $f_{d}$, the complex oscillation amplitude $\widetilde{A}$ can be calculated through

$$
\widetilde{A}(\omega)=h(\omega) F_{d}=\Sigma_{n} \frac{F_{d}}{k_{n}} \frac{1}{\left(1-\omega^{2} / \omega_{r, n}^{2}\right)+i \omega / \omega_{r, n} Q_{n}},
$$

where $\omega=\omega_{d}=2 \pi f_{d}$ is the drive frequency, $F_{d}$ is the driving force amplitude, $Q_{n}$ is the effective quality factor, and $\omega_{r, n}=2 \pi\left(f_{n}+\delta f_{n}\right)$ is the resonance frequency of the mode $n$ perturbed by the tip-sample interaction. The nonlinear tipsample interaction can be modeled using a piecewise description. ${ }^{16}$ In this approach the interaction force $F\left(z_{\mathrm{ts}}\right)$ can be described in terms of tip and sample elastic moduli and tip radius as a function of tip-sample separation $z_{\text {ts. }}$. For the sake of simplicity the nonconservative effects are ignored. For $A_{0} \simeq 10 \mathrm{~nm}$ and $A_{\mathrm{LO}}<1 \mathrm{~nm}$, and assuming that the resulting oscillations at frequencies other than $f_{\mathrm{LO}}$ and $f_{d} \approx f_{0}$ are also smaller than $0.1 \mathrm{~nm}$, the time-dependent tip-sample separation $z_{\mathrm{ts}}(t)$ can be approximated as
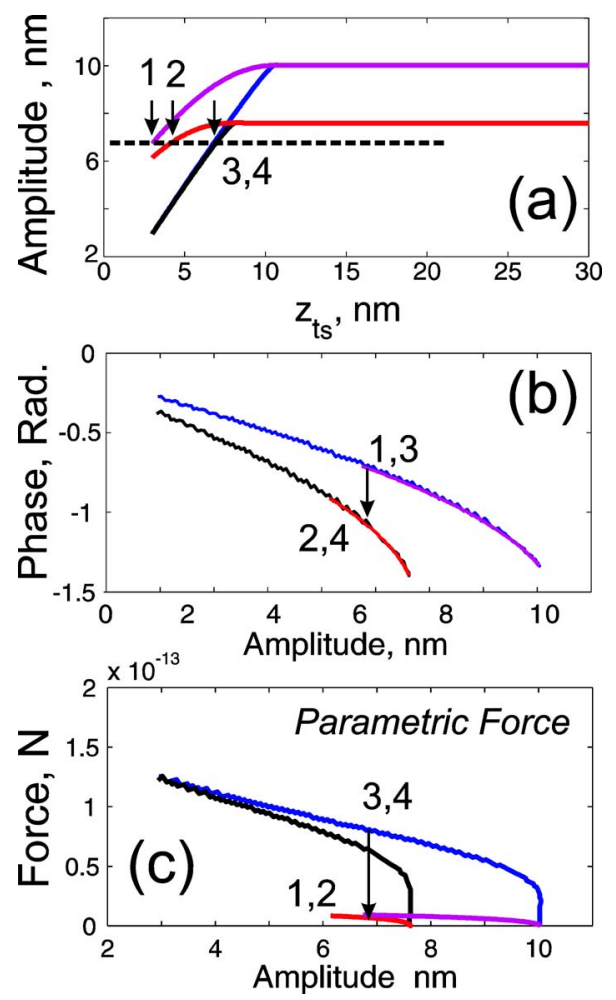

FIG. 2. (Color online) Simulation results for PUC process in the repulsive regime while fundamental mode is used for tapping and second order mode is used for detection. (a) Amplitude vs $z_{\text {ts }}$ for si and PS samples for $Q_{0}$ $=200$ and 150. (b) Phase vs $A_{0}$ shows dissipation contrast. (c) Parametrically up-converted force amplitude calculated for $A_{\mathrm{LO}}=0.2 \mathrm{~nm}$ and $f_{\mathrm{LO}}=f_{1}-f_{0}^{\prime} \mathrm{vs}$ $A_{0}$ shows that contrast is dominated by elasticity of sample.

$$
z_{\mathrm{ts}}(t)=z_{0}+A_{\mathrm{LO}} \cos \left(2 \pi f_{\mathrm{LO}} t\right)+A_{0} \cos \left(2 \pi f_{d} t\right),
$$

where $z_{0}$ is the average tip-sample separation. In order to calculate $A_{0}$ at a given tip-sample separation, Eqs. (1) and (2) are solved iteratively for $\widetilde{A}$ for a given set of parameters and the proper solution is selected. In the analysis, we make the approximation that $A_{\mathrm{LO}}, A_{1} \ll A_{0}$, and higher order parametric processes are ignored (i.e., parametric mixing happens only once). Using Eq. (3) and the interaction model, a timedependent interaction force $F(t)$ can be calculated. The harmonic component of $F(t)$ at frequency $\omega$ can be calculated by $F_{\omega}=\int F(t) \exp (-i \omega t) d t$. The amplitude of oscillation of the cantilever can be then calculated through Eq. (2). Using the described approach, $A_{0}$ versus $z_{0}$ [Fig. 2(a)] and parametrically converted force amplitude $F_{\omega 1}$ versus $A_{0}$ [Fig. 2(c)] are calculated where curves are plotted for silicon $(\mathrm{Si})$, and polystyrene (PS) surfaces. PS is chosen as an example soft material. Since dissipative interactions were not explicitly included in the simulations, in order to observe the effect of dissipation, the calculations are repeated with $Q$ factors of 200 and 150. In tapping mode, feedback fixes $A_{0}$. Therefore, to compare material contrast observed in different channels for a given $A_{0}$ set point, we label the equilibrium points as $1 \doteq(\mathrm{PS}, Q=200), 2 \doteq(\mathrm{PS}, Q=150), 3 \doteq(\mathrm{Si}, Q=200)$, and $4 \doteq(\mathrm{Si}, Q=150)$, respectively. Conventional phase contrast of the tapping mode is seen to depend only on dissipation, as seen in Fig. 2(b). However, parametrically converted forces [Fig. 2(c)] show elasticity dependent contrast.

In order to demonstrate that the material dependent contrast can be imaged using the PUC/PDC processes, images were obtained on polymethyl methacrylate (PMMA) patterns 

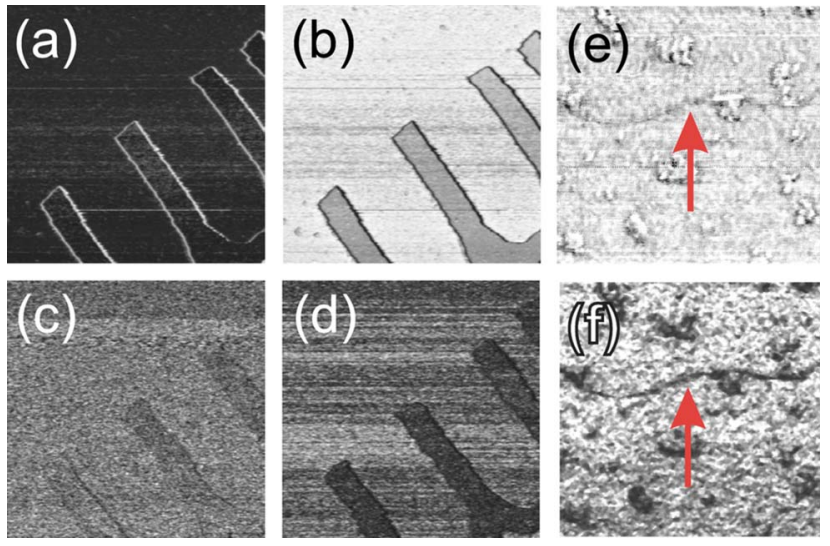

FIG. 3. (Color online) Material contrast observed through PUC in the attractive regime on PMMA patterns on silicon. (a) Phase contrast image. (b) Parametrically up-converted force detected at the second order mode frequency when sample is oscillated at $f_{\mathrm{LO}} \approx f_{1}-f_{0}$, (c) $f_{\mathrm{LO}} \approx f_{1}-2 f_{0}$, and (d) $f_{\mathrm{LO}} \approx f_{1}-3 f_{0}$. Images acquired with $0.5 \mathrm{~Hz}$ per line, $5 \mu \mathrm{m}$ image width. (e) Material contrast can observed through PDC in the repulsive regime with plasmid DNA strands on silicon: Conventional phase (full scale $30^{\circ}$ ) and (f) PDC signal at $90 \mathrm{KHz}$. Double strand (arrow), curled particles, and bundles of DNA can be discerned. Softer material gives darker signature. Images acquired with $1.5 \mathrm{~Hz}$ per line, $0.25 \mu \mathrm{m}$ image width.

on silicon [Fig. 3(a)-3(d)] and on plasmid DNA strands on silicon [Figs. 3(e) and 3(f)]. On large PMMA patterns on silicon, in the attractive regime where there is little dissipation, PUC contrast is observed for the conversion of fundamental and third harmonics [Figs. 3(b) and 3(d)] even when there is no significant phase contrast [Fig. 3(a)]. The absence of dissipation is also directly observed in the low contrast of Fig. 3(c), where the second harmonic of the interaction force is monitored. In the attractive regime, simulations show reduced parametric generation. Therefore, in order to observe the contrast, considerably larger (about a nanometer) $A_{\mathrm{LO}}$ was used. In order to demonstrate applicability of the technique to biological molecules, a sample with DNA strands on silicon was imaged using the second order flexural mode for tapping and using PDC. In this case, repulsive mode is easier to obtain and elasticity dependent PDC contrast can be observed. The observed contrast is parallel with theoretical predictions of Fig. 2(c), where increasing sample modulus results in increased parametric conversion. It was also observed that (data not shown) increasing $A_{\mathrm{LO}}$ or using a much lower amplitude set point results in destruction of isolated DNA strands due to increased peak interaction forces. Simulations show that for standard cantilevers, the interaction pressures are large enough to disturb the molecule's structural integrity. Therefore the technique is applicable for molecular imaging but care must be given to keep the peak forces low.

The method presented here can be applied using a standard rectangular cantilever and a standard AFM system with a vibrating sample holder or an AFM system that allows access to the lever dither piezo by external electronics. In multifrequency excitation through the cantilever dither piezo, the oscillation amplitudes are determined by the lever transfer function, and imaging the fifth harmonic of the force was seen to be most convenient using the PUC process due to enhanced intermediate frequency excitation. Elasticity dependent contrast on molecular level can be observed using a standard cantilever. Further optimization of the cantilever properties can be done to operate the technique with lower interaction forces. The technique presented here can be used to study tip-sample interactions and nanoscale contact mechanics in greater detail than possible with a standard tapping mode AFM. The technique can also potentially be applied for label-free biomolecular detection using integrated micro and nanomechanical devices.

This work was partially funded by Tübitak Grant No. $107 T 547$ and by funding from the State Planning Agency of the Turkish Republic. Aykutlu Dâna thanks Özgür Şahin for enlightening discussions on tip-sample interactions.

${ }^{1}$ G. Binnig, C. F. Quate, and C. Gerber, Phys. Rev. Lett. 56, 930 (1986).

${ }^{2}$ Y. Martin, C. C. Williams, and H. K. Wickramasinghe, J. Appl. Phys. 61, 4723 (1987).

${ }^{3}$ J. Tamayo and R. Garcia, Appl. Phys. Lett. 73, 2926 (1998).

${ }_{4}^{4}$ J. P. Cleveland, B. Anczykowski, A. E. Schmid, and V. B. Elings, Appl. Phys. Lett. 72, 2613 (1998).

${ }^{5}$ R. W. Stark, T. Drobek, and W. M. Heckl, Appl. Phys. Lett. 74, 3296 (1999).

${ }^{6}$ R. W. Stark and W. M. Heckl, Surf. Sci. 457, 219 (2000).

${ }^{7}$ R. W. Stark and W. M. Heckl, Rev. Sci. Instrum. 74, 5111 (2003).

${ }^{8}$ S. Crittenden, A. Raman, and R. Reifenberger, Phys. Rev. B 72, 235422 (2005).

${ }^{9}$ M. Stark, R. W. Stark, W. M. Heckl, and R. Guckenberger, Appl. Phys. Lett. 77, 3293 (2000).

${ }^{10}$ M. Balantekin and A. Atalar, Appl. Phys. Lett. 87, 243513 (2005).

${ }^{11}$ M. Balantekin and A. Atalar, Phys. Rev. B 71, 125416 (2005).

${ }^{12}$ M. Stark, R. W. Stark, W. M. Heckl, and R. Guckenberger, Proc. Natl. Acad. Sci. U.S.A. 99, 8473 (2002).

${ }^{13}$ O. Sahin, C. F. Quate, O. Solgaard, and A. Atalar, Phys. Rev. B 69, 165416 (2004).

${ }^{14}$ N. F. Martinez, S. Patil, J. R. Lozano, and R. Garcia, Appl. Phys. Lett. 89, 153115 (2006).

${ }^{15}$ J. R. Lozano and R. Garcia, Phys. Rev. Lett. 100, 076102 (2008).

${ }^{16}$ R. Garcia and A. San Paulo, Phys. Rev. B 60, 4961 (1999).

${ }^{17}$ O. Morgul, Phys. Lett. A 262, 144 (1999).

${ }^{18}$ F. J. Giessibl, Appl. Phys. Lett. 78, 123 (2001). 